\title{
Multiple Relay Nodes Selection Scheme using Exit Time Variation for Efficient Data Dissemination in VANET
}

\author{
Deepak Gupta ${ }^{1}$, Rakesh Rathi ${ }^{2}$, Shikha Gupta ${ }^{3}$, Neetu Sharma ${ }^{4}$ \\ Department of Computer Science and Engineering \\ Engineering College Ajmer \\ India
}

\begin{abstract}
Efficient Data dissemination in VANET is still the challenge because of variable speed of vehicles, road conditions, frequent fragmentation etc. In this article a selective forwarding data dissemination scheme using exit time differences in vehicles for highway lanes scenario is proposed that focuses on the solution of broadcast storm, less coverage, transmission delay and reliable data delivery. Our approach is selecting multiple forwarding nodes to increase coverage in less delay. In this article road lanes concept is used to identify the moving node direction. Redundant regions and zones technique in proposed approach is reducing the processing of parameters at significant extents. Simulation of proposed approach is done using NS2 and SUMO. Output of implementation is compared with unidirectional flooding, KB_Selective, and LT_Selective techniques. Result analysis shown that the proposed technique is much efficient and it increases the rate of coverage up to $23 \%$. Also it reduces the delay up to $18 \%$ in data delivery ratio. This methodology also improves the performance of system by increasing the throughput and reducing the collision rate in comparison with other methods.
\end{abstract}

Keywords-Broadcasting; disseminations; exit time; highway lanes; relay nodes; vehicle speed; vehicular ad hoc networks

\section{INTRODUCTION}

The networks of moving vehicles are used to disseminate important information among vehicles which includes safety related information, driving support, entertainment, and other mobile commercial services using wireless communication. Communication through vehicular ad hoc network provides the facility of managing and monitoring the traffic services for improvement and maintaining the flow of vehicles on road [1]. VANETs have a fast changing topology where nodes (vehicles) are moving at high and variable speeds in various directions. Such networks are categorized in self-organising networks that can disseminate data with or without requiring any fixed infrastructure [2], [3]. Communication is possible when the vehicles involve in network are differ from normal vehicles. Vehicles in vehicular ad hoc networks are enabled with On Board Unit (OBU). OBU is special hardware in the form of embedded circuit board or ICs that has collection of sensors with processing capabilities to connect the other vehicle in the range. When vehicles are connected to other vehicles then it can transmit or receive the information or vehicles are able to exchange signal and related information in the form of packet transmission [4]. There is another important fixed unit along road side named as Road Side Unit or RSU. When RSU communicate with vehicles through their OBU then this is vehicle to infrastructure (V2I) communication. RSU temporarily stores the details of all vehicles in its range. This unit can connect to cloud, fog or other internet servers to send and receive information to and from other systems as per requirement. This is Infrastructure to Infrastructure (I2I) communication [5]. Periodic beacons are used to exchange the traffic-related information with OBU to aware the drivers of other connected vehicles for traffic and other related conditions [6]. RSUs also exchange information periodically for updating databases. As vehicles have radar at both front and rear side so it can communicate with vehicles moving in any directions [7]. Alert can be seen on display units or can be felt through vibrations, sounds or any other type of alarming options [8]. Fig. 1 is showing the typical VANET scenario consists of OBU enabled vehicles, RSUs, Cloud or other internet server. Remaining article is arranged as follows: Section 2 contains brief overview about data dissemination. In Section 3 the related work is explained followed by proposed methodology in Section 4. Section 5 includes experimental setup and comparison of results with existing schemes. Sections 6 and 7 presents conclusion and future scope of our approach.

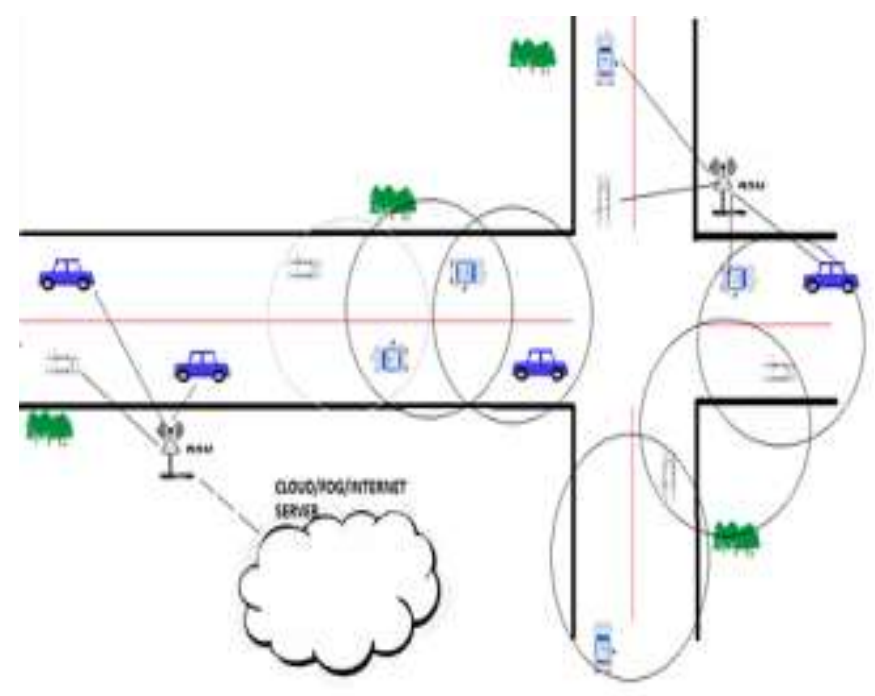

Fig. 1. Basic Vehicular ad HOC Network Scenario. 


\section{VANET DATA DISSEMINATION}

As in Vehicle to Vehicle communication, vehicles are moving with variable speeds and directions, it is very difficult task to deliver data at proper time that is, the time when the data is valued for receiver for example there is no use of information received by a vehicle user regarding traffic support like jam due to road accident when vehicle already reached there and hang up in traffic [9]. This is the problem of discovering and distributing information quickly to nodes. Data dissemination is challenging because vehicular network changes rapidly, variables speed vehicles, different road conditions, frequent fragmentation or others parameters [10]. The process of delivering the data or information to other vehicles or nodes in distributed wireless network is called as data dissemination. Proper dissemination schemes are helpful in delivery of data to desired number of vehicles at in proper time [11]. This also helps in reducing the data congestion and traffic. Different characteristics of vehicular ad hoc networks like variable speeds, types of mobility models, communication flow etc. creates networking complications, which requires the solution for efficient and effective dissemination protocol [12]. Vehicles create a dynamic scenario due to variation in speed and therefore very short life span in several lanes. Many researchers proposed various schemes for improving the dissemination process between vehicles that can be categorized as infrastructure, broadcast and geocast [13]. RSU is basic requirement for infrastructure based schemes. In broadcast based the sender forward information to all nodes in network while in geocast, nodes are belongs to zone of relevance [14]. Some basic data dissemination schemes are shown in Fig. 2.

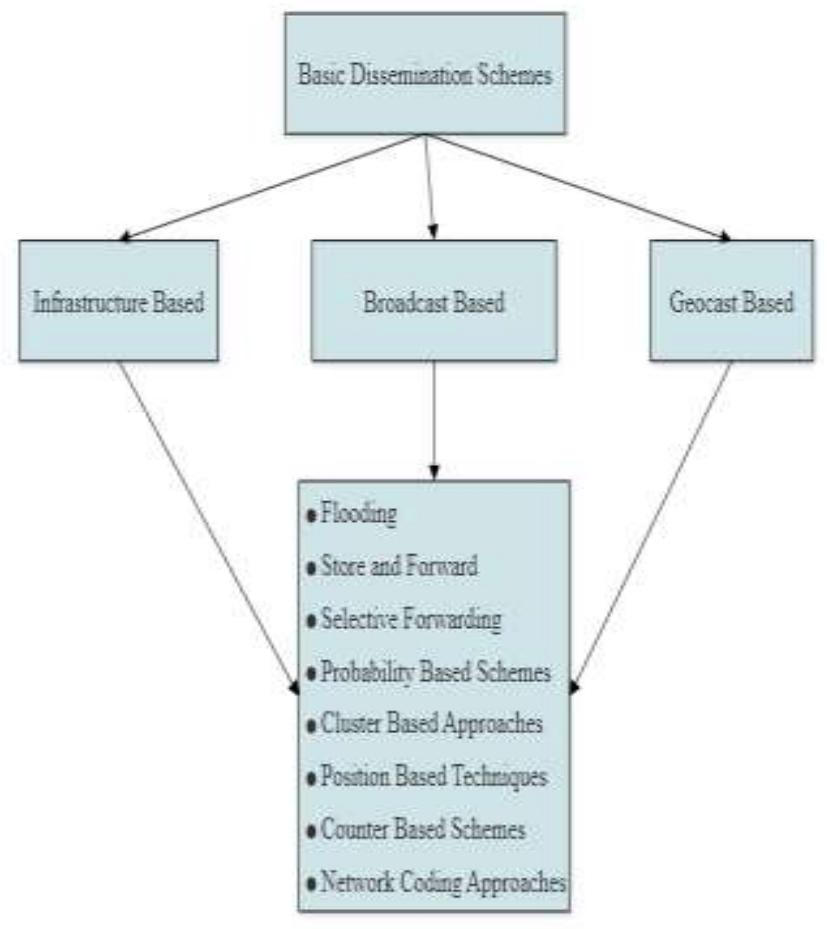

Fig. 2. FBasic Dissemination Schemes in VANET.
Broadcasting the information for dissemination in vehicles may create the problems like broadcast storms and network congestion. Another problem that arise during VANET data dissemination is the disconnected networks problem which mostly occurs in areas having sparse traffic that is, less number of connected vehicles are available for information transfer [15]. This problem of disconnected network may leads to loss of data or information before reaching to actual receiver or desired number of receivers. Many researchers worked on these problems and proposed solutions. We are discussing some in next section of the article.

\section{RELATED WORK}

Tian et al. [16] introduced Traffic Adaptive Data Dissemination (TrAD) Protocol that defines concept of a directional cluster which includes the vehicles around the sender whose direction of movement is same as of sender. There is a coordinator in the cluster which is the vehicle or node at the intersection whereas breaker is the vehicle which is about to leave the cluster. This scheme was supporting broadcast storm reduction and based on forwarding technique but there was more delay due to high processing at the intersections of roads in urban scenarios. Geocast based Information - Centric Opportunistic Data Dissemination scheme was proposed by Leal et al. [17] which classifying messages as periodic beacons and event driven messages named as Cooperative Awareness Messages (CAM) and Decentralized Environmental Notification Messages (DENM). In this the probability of retransmission of a message keeps on decreasing with distance of the vehicle from the event location. Density-based Gossiping protocol tries to address an issue of Geographic Gossiping Protocol which was not as much supportable as proposed in areas with non-uniform distribution of vehicles. Nikolovski et al. [18] proposed Delay Tolerant and Predictive protocol (DTP-DDP) of Dissemination, focused mainly to overcome from broadcast storm problem by reducing number of rebroadcasting. Message strength or power was computed for every received message and if it is less than $12 \%$ then it immediately rebroadcast the message, assuming that it is very far from sender or it may be at transmission boundary of sender. For message having power more than the threshold, decision was based on distance and direction of receiver node with respect to event location which involved lots of processing and computing. This may produce delay in further broadcasting of information. Retransmission decision based on number of times of message received by node, in defined time duration is introduced by Bakhouya et al. [19] named as adaptive and decentralized approach (AID). Assumption behind this was that, in case of dense network of vehicle message can be forwarded from many other vehicles, so better to drop the message instead of further forwarding. This theory will help in reducing the congestion in network in dense network but not as much effective as proposed in sparse network due to overhead created for retransmission decision. Costa et al [20] introduced beacon based DDRX protocol for data dissemination in urban scenario that is depends on vehicle network density for TMS related applications claiming in 
overhead reduction and less delay by creating a graph of connected vehicles up to 2 -hop network. To have knowledge of 2-hop vehicles before transmission of information may produce delay which can be high in urban traffic. Ali et al. [21] introduced an infrastructure less scheme for data broadcasting was fully based on position of vehicles and clusters for emergency messages in high dense scenarios of vehicles. Vehicle's information like positions, speed and moving direction was exchanged using periodic beacons signals. Based on these parameters a node may be the cluster member or not. If a node was cluster member then only it would be the part of relay node selection process otherwise not. In distributed traffic management systems, Costa et al. [22] introduce a protocol for efficient data dissemination by selecting the best relay node for sending traffic management services messages to cover maximum nodes with less overhead and transmission delay. In selecting the relay node every vehicle must have knowledge of 1 and 2-hop neighbours, this may introduce delay in coverage. A probability based broadcasting approach for safety related messages was given by Sospeter et al. [23] named as Effective and Efficient Adaptive Probability Data (EEAPD) Dissemination. Information forwarder decision was based on the vehicle (source node) to vehicle distance, vehicle density and direction of message. Relationship between number of vehicles (vehicle density) and particular road segment is defined by a metric called redundancy ratio which was not highly effective in urban scenarios. Adaptive Data Dissemination Protocol (AddP) was introduced by Oliveira et al. [24], focusing on reliability of safety message dissemination. It was a multi-hop broadcast protocol where rebroadcasting was reduced through aggregation of data and network coding. A relay or forwarding node selection was based on local vehicle density and distance (from neighbour's nodes) involves huge processing at each node and may led to delay in dense networks. Baiocchi et al. [25] proposed TimerBased Backbone Network (TBN) protocol. Forwarding or Rebroadcast decisions were taken using Monte Carlo algorithm for randomization method. As the approach was beacon-less and time based, therefore there were chances to not forward important information due to network delay or other after timer expired. Liu et al. [26] proposed an approach based on maximum flooding. This was an effective solution towards data dissemination but cannot handle the dense network due to broadcast storm as the focus was on mostly on maximum coverage. Chaqfeh et al. [27] proposed another approach to solve the congestion problem in heavy traffic during data transmission among vehicles, based on the concept of relay node selection but they have still not considered some other parameters which can solve the problem of broadcast storm more efficiently. Further the technique based on probability during flooding was proposed by Gutiérrez-Reina et al. [28] for data dissemination in VANET, but the problem of delay in coverage was still persists and overall throughput was not up to the mark as proposed in dense network due to less rate of coverage. Qureshi et al. [29] proposed cluster based approach for vehicular data dissemination. Making clusters of networks to process the information for dissemination was one of the efficient technique but to handle the cluster members and other overheads there is quite more processing was required on account of which an important and urgent information may have to suffer. With this survey we found that still the speed and lane of vehicles were rarely used by researchers which could be important parameters in dissemination process. These parameters may have important role in solving the problems or issues by broadcast storm like coverage, delay, collision etc. In section 4, we are proposing an effective solution in such relay networks where information from one network, is pass on to another using relay nodes. We have used speed as important parameter that covers some technical gaps and problems in relay node selection or selective forwarding approaches.

\section{PRoposed Methodology}

In the highway scenario of vehicular ad hoc communication, number of lanes is an important parameter that can be used in assignment of direction of moving vehicles and helps in the process of relay nodes selection effectively. Number of lanes, $L$ on highway or highway lanes may vary as per the regions, states and countries, and always are even in number as in (1).

$$
\text { Lanes }=\{L:[(L \leq 16) \text { and }((L \bmod 2)==0)]\}
$$

In our proposed technique we are assuming maximum 16 lanes. Periodically values of basic parameters like position, speed and lane number for every vehicles in 1-hop will exchange with each other. Nodes maintain the dynamic record of received coordinates of neighbor's and start the grouping of these records into the same or other direction vehicles with respect to itself. It has been assumed that vehicles on highway are either moving from left to right or right to left. Depending on direction of moving of source vehicles $\left(N_{\text {SOURCE }}\right)$, other nodes in the transmission range of source will considered as same or opposite direction moving vehicles. Fig. 3 shows the vehicles scenario of proposed 1-hop neighbors of $N_{\text {SOURCE }}$ (black car moving right) and respective mapping to regions according to moving directions in 8 lanes.

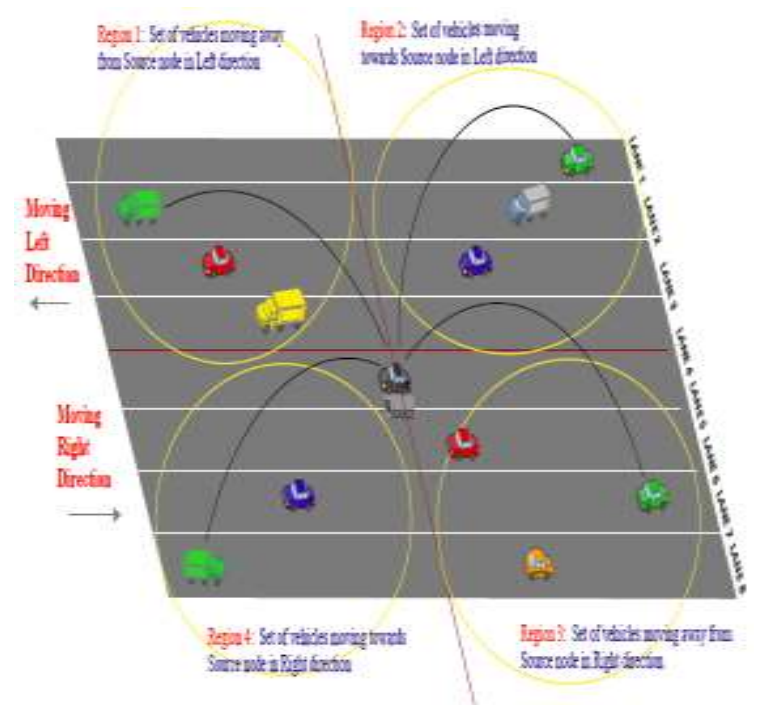

Fig. 3. Directions and Regions Mapping with respect to Source (Black Car). 
If source is moving left then the defined procedures in later subsections of this article will change accordingly. We are separating all 1-hop neighbors into four regions that is, region 1 , region 2, region 3 and region 4 with respect to source node.

\section{A. Direction Assignment Process (DAP)}

Direction assignment process of proposed technique is assigning directions with respect to source vehicle for each 1hop vehicles in source transmission range; procedure Direction_Assign doing the work of direction assignment at $N_{\text {SOURCE }}$ using the exchanged records of parameters.

Procedure: Direction_Assign (NLIST, L_ID, L)

Input: 1-hop Node list $\left(N_{L I S T}\right)$ in source
transmission range, Lane number of each
neighbor $\left(L_{-} I D\right)$, Number of lanes on
highwy $(L)$
Output: Assignment of directions to 1-hop nodes
1. for each $\left\{N i \in N_{L I S T}\right\}$ do
2. if $L_{-} I D<=|L / 2|$
3. ML[ $\leftarrow N_{i} / /$ Moving Left Assignment
4. otherwise,
5. MR[ $\leftarrow N_{i} / /$ Moving Right Assignment
6. return $\{M L[], M R[]\}$

After assignment of moving direction and using the position of vehicles, source node divides the ranged vehicles into the set of same and opposite directions from itself; having confidence in that the vehicles moving away from the source will provide faster coverage as compared to the vehicles moving towards source. Keeping this belief and to reduce processing, vehicles are divided into Rejection and Selection zone. Vehicles belongs to rejection zone will not include in the selection process for relay candidate. Flow of working approach is shown in Fig. 4.

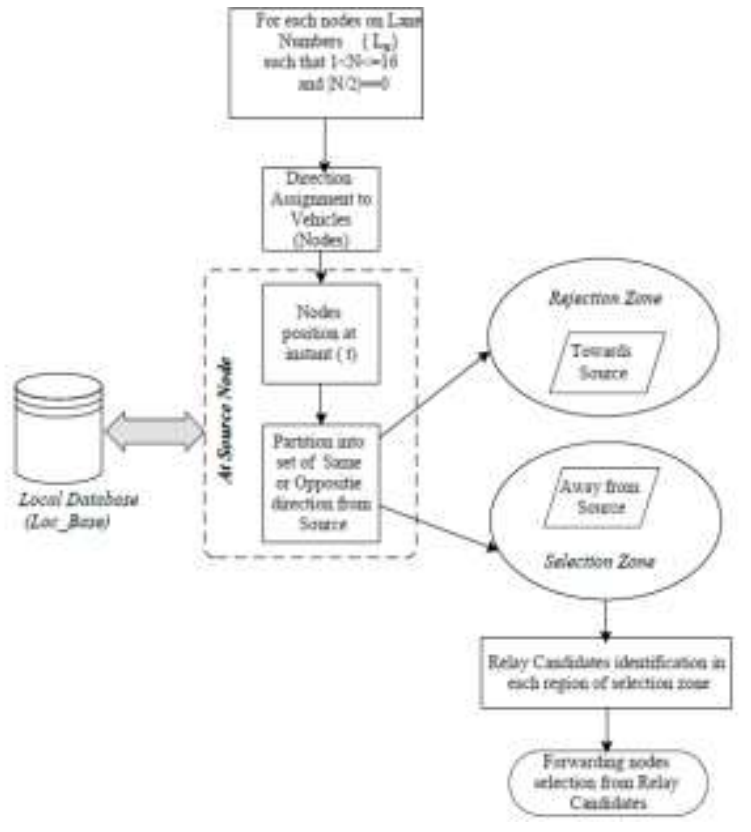

Fig. 4. Flow of Proposed Working Approach.

\section{B. Region Assignment Process (RAP)}

After assigning the direction of moving of nodes at $N_{\text {SOURCE }}$, region assignment of vehicles is performed. Various regions for proposed approach are defined as follows:

Region $1 \in$ Vehicles moving left and away from $N_{\text {SOURCE. }}$

Region $2 \epsilon$ Vehicles moving left and towards from $N_{\text {SOURCE }}$.

Region $3 €$ Vehicles moving right and away from $N_{\text {SOURCE }}$.

Region $4 \mathbf{\epsilon}$ Vehicles moving right and away from $N_{\text {SOURCE. }}$

Assignment of each 1-hop vehicle to respective region with respect to source is carried out by below procedure.

Procedure (at $\left.\mathrm{N}_{\text {SOURCE }}\right)$ : Region_Assign $\left(N_{\text {LIST }}\right)$

\begin{tabular}{|c|c|}
\hline $\begin{array}{l}1 . \\
2 .\end{array}$ & $\begin{array}{l}\text { for each } N \text { in } N_{\text {LIST }} \\
\quad \text { if LOC_Base }\left(N_{\text {LOCATION }}\right) \boldsymbol{\epsilon}\left[\left(X<X_{\text {SOURCE }}\right) \text { AND }\right. \\
\left.\left(Y>Y_{\text {SOURCE }}\right)\right]\end{array}$ \\
\hline 3. & Region1[ ] $\leftarrow N$ \\
\hline 4. & A1_N $N_{\text {SOURCE }}[\quad] \leftarrow N$ \\
\hline 5. & $\begin{array}{l}\text { else if LOC_Base }\left(N_{\text {LOCATION }}\right) \in\left[\left(X>X_{\text {SOURCE }}\right)\right. \\
\left.\operatorname{AND}\left(Y>Y_{\text {SOURCE }}\right)\right]\end{array}$ \\
\hline 6. & Region2[ ] $\leftarrow N$ \\
\hline 7. & $T 1 \_N_{\text {SOURCE }}[\quad] \leftarrow N$ \\
\hline 8. & $\begin{array}{l}\text { else if LOC_Base }\left(N_{\text {LOCATION }}\right) \in\left[\left(X>X_{\text {SOURCE }}\right)\right. \\
\left.\text { AND }\left(Y<Y_{\text {SOURCE }}\right)\right]\end{array}$ \\
\hline 9. & Region3 []$\leftarrow N$ \\
\hline 10. & A2_ $N_{\text {SOURCE }}[\quad] \leftarrow N$ \\
\hline 11. & 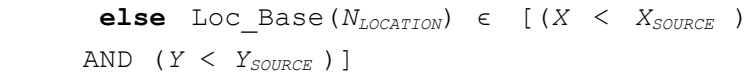 \\
\hline 12. & Region4[ ] $\leftarrow N$ \\
\hline 13. & 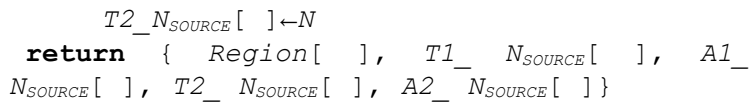 \\
\hline
\end{tabular}

\section{Zones Selecting and Rejecting Process (ZSRP)}

Next to RAP for 1-hop moving vehicles with respect to source the system proceed to select the best relay node. In our work we are selecting two relay nodes instead of one to cover maximum area in less time. For this we are considering an important parameter that is speed of moving vehicles. This parameter is not focused in most of the earlier researches. In this approach with the help of speed of moving vehicle we are efficiently selecting the forwarder nodes from selection zone. In almost all previous approaches the farthest node from source was considered as the relay node but here we are also considering the node just behind the farthest node in both directions. It could possible that the node just behind farthest node may leave the transmission range of source first because of speed difference, it may overtake farthest node within the range itself.

This leave time computation can applied to all the nodes in selection zone but we are not calculating that, assuming that there is less probability of existence of node which have less leave time in comparison with farthest or behind farthest node. 
Also if such vehicle exists then also the coverage by farthest or behind farthest node will be more as these nodes will be in early contact to 2 - hop nodes.

Including computation of leave time for each node will increases overhead and processing which may introduce delay in coverage. Fig. 5, showing the representation of such nodes on x-y coordinate system. Here we are assuming that $N_{\text {SOURCE }}$ is at $(0,0)$ in red colour. Green nodes are the relay candidates and their remaining distances from source are represent by Rld1, Rld2 in region 1 while in region 3 they are represents by $R 3 d l$ and $R 3 d 2$. Vehicles in region 2 and region 4 belongs to rejection zone, so they are not considered for relay candidate and marked in black colour. Following possible cases occurs for rejection zone vehicles according to the source moving direction as in (2) and (3).

Case I: $\left[\left(\operatorname{dir}\left(N_{\text {SOURCE }}\right)=M R\right) \wedge(\operatorname{pos}(N) \varepsilon((x, y) \vee(-x,-y)))\right]$

Description: If any vehicle selected as forwarding node and belong to either $(-x,-y)$ or $(x, y)$ region when source moving towards positive $x$, then it would covers mostly those vehicles which are already in the range of source only. Node selected as relay, will forward the information as soon as it received and to cover the region outside the source transmission range will take some time by such nodes even having the minimum time of leave $(t t l)$.

Case II: $\left[\left(\operatorname{dir}\left(N_{\text {SOURCE }}\right)=M L\right) \wedge(\operatorname{pos}(N) \varepsilon((x,-y) \vee(-x, y)))\right]$

Description: If any vehicle selected as forwarding node and belong to either $(-x, y)$ or $(x,-y)$ region when source moving towards negative $x$, then also it covers mostly the vehicles which are in the range of source and have already the information.

Remaining distance, let for green node in Fig. 6, is $\left|\mathrm{AC}=\mathrm{BD}=R_{K} d_{K}\right|$ in region $K$. Position of this node is represents as $[(-x, y) \sim|x|,|y|)]$. Transmission range of source $(T)$ creating a circle of radius $T$. Also $O D=|x|$ and $C D=A B=|y|$. Therefore value of $R_{K} d_{K}$ and remaining time to leave ( $\left.t t l\right)$ from source range are computed using (4) and (5) respectively.

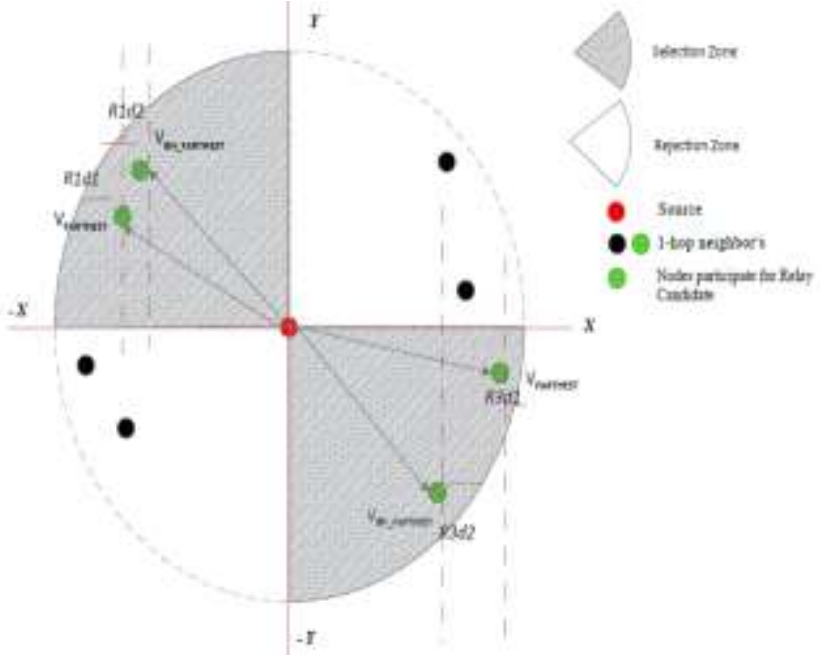

Fig. 5. Representation of 1-hop Nodes on x-y Coordinate System.

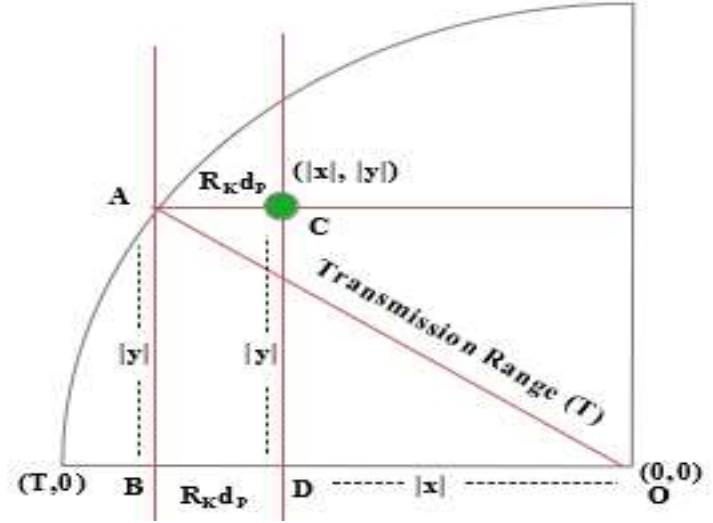

Fig. 6. Computation of Remaining Span from Source Transmission Range.

$$
\begin{aligned}
& R_{K} d_{P}=\left\{\left(\sqrt{T^{2}-(|y|)^{2}}\right)-|x|\right\} \\
& t t l[]=\left\{\left(R_{K} d P\right) *\left(\left[V_{\text {FARTHEST }}\right] \operatorname{or}\left[V_{B H_{-}} \text {FARTHEST }\right]\right){ }_{K}\right\}
\end{aligned}
$$

$\mathrm{K}$ is region number and $P \varepsilon\{$ FARTHEST,BH_FARTHEST $\}$

\section{Relay Nodes Selection Process (RNSP)}

This subsection is explaining the process vehicle detection which will cover the next network earliest. RNSP is selecting two relay or forwarder nodes: relay_node1 and relay_node 2 in opposite directions from selection zones. The procedure is considering both farthest and behind the farthest vehicles in selection zones based on the assumption that the behind vehicle may leave the range first covering more vehicles outside the range of source in less time. So the aim of RNSP is vehicle detection which will leave the transmission range of source early between farthest node and behind the farthest node, in both regions having vehicles moving away from source. For this leave time calculation, speed and distance between these vehicles are obtained using global positioning system and other parameters exchanged periodically and stored in local database of nodes. Fig. 7 shows the steps of RNSP procedure.

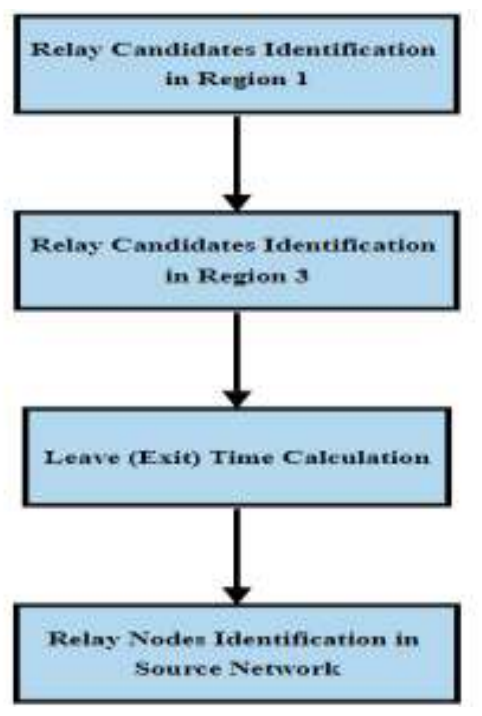

Fig. 7. Steps for RNSP. 


\section{Algorithm: RN_Election}

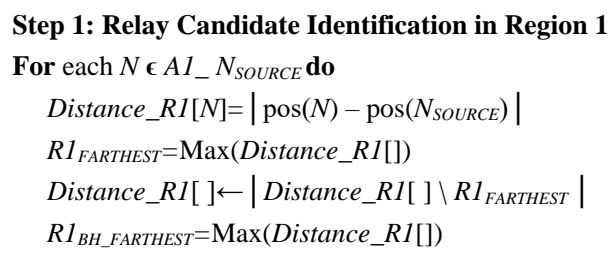

Step 2: Relay Candidate Identification in Region 3

For each $N \in A 2 \_N_{S O U R C E}$ do

Distance_R2 $[N]=\left|\operatorname{pos}(N)-\operatorname{pos}\left(N_{\text {SOURCE }}\right)\right|$

$R 2_{\text {FARTHEST }}=\operatorname{Max}($ Distance_R2[])

Distance_R2[] $\leftarrow \mid$ Distance_R2[] ]R2 $2_{\text {FARTHEST }} \mid$

$R 2_{B H_{-} \text {FARTHEST }}=\operatorname{Max}($ Distance_R2[])

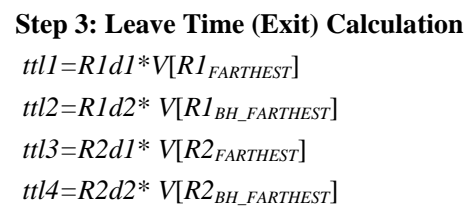

Step 4: Relay Nodes Identification in Source Network if $t t 1<=t t l 2$

$$
\begin{aligned}
& \text { relay_node } 1 \leftarrow R 1_{\text {FARTHEST }} \\
& \text { else } \\
& \text { if } t t l 3<=t t l 4 \\
& \text { relay_node } 1 \leftarrow R 1_{\text {BH_FARTHEST }} \\
& \text { relay_node } 2 \leftarrow R 2_{\text {FARTHEST }} \\
& \text { relay_node } 2 \leftarrow R 2_{\text {BH_FARTHEST }}
\end{aligned}
$$

\begin{tabular}{|c|c|}
\hline Notations & Interpretation \\
\hline $\mathrm{L}$ & Number of Lanes \\
\hline $\mathrm{V}$ & Vehicle speed \\
\hline $\mathrm{t}$ & Time instant \\
\hline L_ID & Unique identification number of lane \\
\hline $\mathrm{N}_{\text {SOURCE }}$ & Source node or vehicle \\
\hline $\mathrm{N}_{\text {LIST }}$ & 1-hop neighbors list of $\mathrm{N}_{\text {SOURCE }}$ \\
\hline MR[ ] & Set of Nodes moving right with respect to $\mathrm{N}_{\text {SOURCE }}$ \\
\hline ML[ ] & Set of Nodes moving left with respect to $\mathrm{N}_{\text {SOURCE }}$ \\
\hline Loc_Base & Local database of each node \\
\hline T1_ N $_{\text {SOURCE }}[]$ & Set of vehicles from region 2 , moving towards $\mathrm{N}_{\text {SOURCE }}$ \\
\hline T2_ $\mathrm{N}_{\text {SOURCE }}[]$ & Set of vehicles from region 4 , moving towards $\mathrm{N}_{\text {SOURCE }}$ \\
\hline $\mathrm{A} 1 \mathrm{~N}_{\text {SOURCE }}[]$ & $\begin{array}{l}\text { Set of vehicles from region 1, moving away from } \\
\mathrm{N}_{\text {SOURCE }}\end{array}$ \\
\hline $\mathrm{A} 2 \_\mathrm{N}_{\text {SOURCE}}[]$ & $\begin{array}{l}\text { Set of vehicles from region 3, moving away from } \\
\mathrm{N}_{\text {SOURCE }}\end{array}$ \\
\hline Distance_R1[] & Distance of all nodes $\epsilon$ Region 1 from $\mathrm{N}_{\text {SOURCE }}$ \\
\hline Distance_R2[] & Distance of all nodes $\epsilon$ Region 3 from $\mathrm{N}_{\text {SOURCE }}$ \\
\hline R1 $1_{\text {FARTHEST }}$ & Farthest vehicle in region 1 from $\mathrm{N}_{\text {SOURCE }}$ \\
\hline
\end{tabular}

\section{E. Notation List}

Table I is showing the list of notations and their interpretation used in our proposed methodology.

TABLE I. NOTATIONS LIST

\begin{tabular}{|l|l|}
\hline $\mathrm{R} 1_{\text {BH_FARTHEST }}$ & Vehicle behind $\mathrm{R} 1_{\text {FARTHEST }}$ \\
\hline $\mathrm{R} 2_{\text {FARTHEST }}$ & Farthest vehicle in region 3 from $\mathrm{N}_{\text {SOURCE }}$ \\
\hline $\mathrm{R} 2_{\text {BH_FARTHEST }}$ & Vehicle behind $\mathrm{R} 2_{\text {FARTHEST }}$ \\
\hline $\mathrm{R} 1 \mathrm{~d} 1$ & Span remains to exit from source range by $\mathrm{R} 1_{\mathrm{FARTHEST}}$ \\
\hline $\mathrm{R} 1 \mathrm{~d} 2$ & Span remains to exit from source range by 1 $_{\text {BH_FARTHEST }}$ \\
\hline $\mathrm{R} 2 \mathrm{~d} 1$ & Span remains to exit from source range by $\mathrm{R} 2_{\mathrm{FARTHEST}}$ \\
\hline $\mathrm{R} 2 \mathrm{~d} 2$ & Span remains to exit from source range by $\mathrm{R} 2_{\text {BH_FARTHEST }}$ \\
\hline $\mathrm{tt} 1$ & Remaining time to exit from source transmission range \\
\hline
\end{tabular}

\section{Performance Evaluation}

\section{A. Simulation Environment}

Proposed work is carried out in NS-2 environment with the supporting tools SUMO and MOVE. For comparing and analysis of results we have assigned the speed randomly to moving vehicles in the range of $20 \mathrm{mph}$ to $80 \mathrm{mph}$. For better analysis we are taking the average of values obtained in 10 simulations. Various simulation parameters taken are shown in Table II. Work has been extended to real time highway fragment of radius $3 \mathrm{~km}$ using OpenStreetMap. Working approach has been examined in the terms of simulation duration and rate of flow of vehicles or vehicle density. Result of proposed work is compared with selective flooding, unidirectional flooding and selective forwarding proposed by Farooq et al. [30] and Pradhan R. [31]. Farooq et al. proposed unidirectional flooding and selective flooding based on knapsack problem (KB_Selective) of weight and profit assignment for optimal (maximum profit) solution. Pradhan R. proposed selective dissemination technique based on leave time (LT_Selective) calculation of nodes to select the forwarding node.

\section{B. Metrics}

- Receiver Ratio $(R R)$ : Receiver ratio in (6) is the ratio of number of nodes received $\left(N_{R}\right)$ the message transmitted by source to the total number of nodes $(N)$ available in the region of interest for $n$ simulations. More the value of this means more the coverage of nodes on highway.

$R R=\left(\sum_{s=1}^{n} \frac{N_{R}}{N}\right) / n$

- Packet Delivery Ratio (PDR): This metric supports the trustworthiness of network and defined as the total number of packets successfully delivered from the total packets transmitted in source node range. High the PDR produces the reliability of network and belief on moving nodes.

$$
A v g_{-} P D R=\left(\sum_{s=1}^{n} \frac{P_{R}}{P_{R}+P_{L}}\right) / n
$$

- Wait Time (WT): Wait time is defined as the amount of time taken by transmitted message $(D)$ to reach at every node in the region. High waiting time is not acceptable in network for important and time bound information.

$$
A v g_{-} W T=\left(\sum_{s=1}^{n}\left(\sum_{i=1}^{N_{R}}\left(D_{i}\right)\right) / N_{R}\right) / n
$$


- Collision Ratio $(C R)$ : Collision ratio is the total number of collided packets $\left(P_{C}\right)$ to the total number of nodes in the network for given duration of time.

$A v g_{-} C R=\left(\sum_{s=1}^{n}\left(\left(P_{C} / N\right)\right)\right) / n$

- Throughput: This parameter is used to find the efficiency of network by computing the size of total message received $(R)$ per unit time as shown in (10).

Throughput $=\left(\sum_{i=1}^{t} R / t\right)$

\section{Simulation Parameters}

The simulation parameters and their corresponding values considered for proposed scheme are listed below in Table II.

TABLE II. PARAMETERS USED FOR SIMULATIONS

\begin{tabular}{|l|l|}
\hline Item & Values \\
\hline Distance & $3 \mathrm{~km}$ \\
\hline Number of lanes & 8 \\
\hline Simulation time & 300 seconds \\
\hline Number of simulations & 10 \\
\hline Vehicle speed & {$[20-80] \mathrm{mph}$} \\
\hline Transmission range & $400 \mathrm{~m}$ \\
\hline Message size & $1 \mathrm{~KB}$ \\
\hline MAC Layer & $802.11 \mathrm{p}$ \\
\hline Traffic rate & {$[100-400]$ vehicles/hr } \\
\hline Simulation framework & NS 2.35 \\
\hline Mobility Simulator & SUMO 0.32 \\
\hline Map tool & OpenStreetMap \\
\hline
\end{tabular}

\section{Simulation Results}

This sub-section includes the results and analysis of simulation based on various parameters as we discussed earlier. Fig. 8 to 12 shows the effect on parameters behaviour with the simulation time by keeping maximum constant number of vehicles (300) in simulation for 300 seconds. As the proposed approach selecting two relay nodes in each direction of moving therefore we are considering half of total vehicles moving left and remaining half are moving right. That means 150 vehicles in each direction.

Table III represents the values of metrics with simulation time and comparison graphs for same are shown in Fig. 8-12. In the beginning of simulation less number of packets generated for transmission and hence most of them are successfully delivered to destination nodes but as the time increases more number of packets are transmitted which increases the collision and congestion. The variation in packets delivery is shown in Fig. 8. Results show that proposed technique is doing far better in comparison to others.

Variation in throughput at early phase of simulation is not changing rapidly. As the time increases it decreases speedily in each round of simulation. This is due to decrease in packet delivery and increases in retransmission. Fig. 9 shows the performance for throughput and it is found that proposed approach is giving better results in comparison to others.

Fig. 10 shows the result of coverage of information among nodes. Comparing to others proposed method is covering the network in approximately $4 \mathrm{~min}$, while the others are covering up to $80 \%$ to $90 \%$ region in this duration. Though the vehicle density is constant and collisions are increasing the coverage is still reaching to $100 \%$ because of packets delivery to some nodes and such nodes are further applying the approach to find the forwarding nodes. These selective forwarders increase the coverage and also transmit message to the nodes which haven't received the packets in previous transmission.

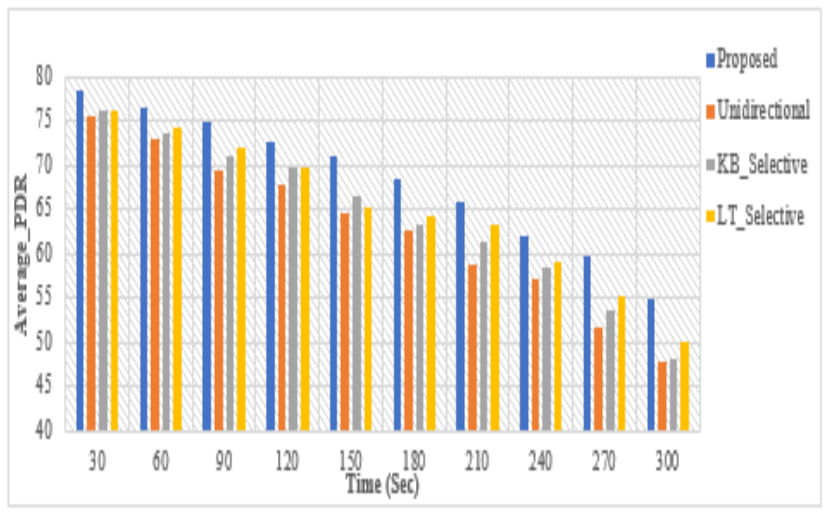

Fig. 8. Simulation Result for Packet Delivery Ratio versus Time.

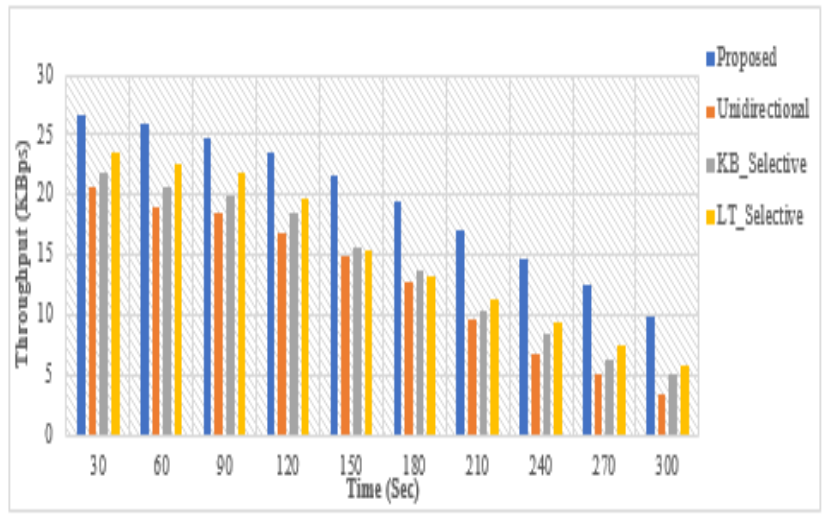

Fig. 9. Simulation Result for throughput versus Time.

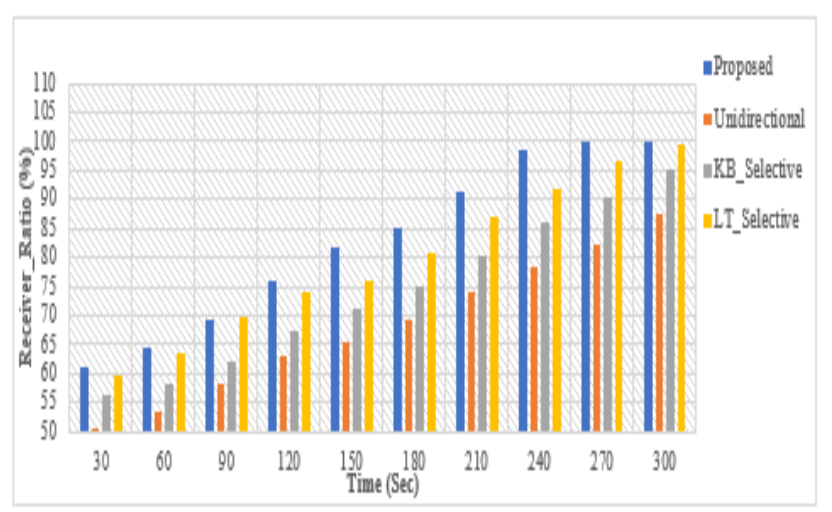

Fig. 10. Simulation Result for Receiver Ratio versus Time. 
TABLE III. RESUlt TABLE WITH SimULATION TIME

\begin{tabular}{|c|c|c|c|c|c|c|c|c|c|c|c|}
\hline \multicolumn{2}{|l|}{ Time (Sec) } & 30 & 60 & 90 & 120 & 150 & 180 & 210 & 240 & 270 & 300 \\
\hline \multirow{4}{*}{ PDR } & Proposed & 78.532 & 76.547 & 75.006 & 72.637 & 70.837 & 68.541 & 65.903 & 62.041 & 59.821 & 54.971 \\
\hline & Unidirectional & 75.554 & 72.827 & 69.214 & 67.803 & 64.443 & 62.53 & 58.71 & 57.196 & 51.62 & 47.645 \\
\hline & KB_Selective & 75.981 & 73.443 & 71.106 & 69.719 & 66.369 & 63.237 & 61.264 & 58.304 & 53.71 & 48.064 \\
\hline & LT_Selective & 76.054 & 74.106 & 72.083 & 69.54 & 65.112 & 64.224 & 63.12 & 59.17 & 55.34 & 50.146 \\
\hline \multirow{4}{*}{$\begin{array}{l}\text { Throughput } \\
\text { (KBps) }\end{array}$} & Proposed & 26.78 & 25.96 & 24.85 & 23.57 & 21.73 & 19.42 & 17.05 & 14.56 & 12.41 & 9.75 \\
\hline & Unidirectional & 20.75 & 19.06 & 18.51 & 16.87 & 14.92 & 12.78 & 9.72 & 6.67 & 4.96 & 3.37 \\
\hline & KB_Selective & 21.96 & 20.77 & 20.06 & 18.41 & 15.71 & 13.64 & 10.32 & 8.51 & 6.33 & 4.97 \\
\hline & LT_Selective & 23.48 & 22.69 & 21.87 & 19.68 & 15.38 & 13.23 & 11.22 & 9.28 & 7.41 & 5.79 \\
\hline \multirow{4}{*}{$\begin{array}{l}\text { Receiver_Ratio } \\
(\%)\end{array}$} & Proposed & 61.219 & 64.296 & 69.451 & 76.145 & 81.781 & 85.116 & 91.407 & 98.391 & 100 & 100 \\
\hline & Unidirectional & 50.612 & 53.437 & 58.295 & 62.805 & 65.457 & 69.421 & 74.103 & 78.306 & 82.116 & 87.362 \\
\hline & KB_Selective & 56.221 & 58.234 & 62.143 & 67.224 & 71.361 & 75.104 & 80.224 & 85.849 & 90.244 & 95.361 \\
\hline & LT_Selective & 59.753 & 63.68 & 69.758 & 73.842 & 75.886 & 80.731 & 87.221 & 91.784 & 96.576 & 99.748 \\
\hline \multirow{4}{*}{$\begin{array}{l}\text { Collision_Ratio } \\
\text { (Avg) }\end{array}$} & Proposed & 0 & 0.4 & 1.1 & 1.8 & 2.5 & 3.2 & 4.2 & 5.8 & 8.6 & 11.4 \\
\hline & Unidirectional & 0 & 0.6 & 1.4 & 2.4 & 3.2 & 5.4 & 6.2 & 8.5 & 12.9 & 16.3 \\
\hline & KB_Selective & 0 & 0.9 & 1.8 & 2.6 & 3.5 & 5.8 & 6.4 & 8.7 & 11.7 & 15.9 \\
\hline & LT_Selective & 0 & 0.7 & 1.4 & 2 & 2.8 & 4.1 & 5.8 & 7.2 & 10.4 & 15.3 \\
\hline \multirow{4}{*}{$\begin{array}{l}\text { Wait_Time } \\
\text { (Avg_Delay) (ms) }\end{array}$} & Proposed & 92.812 & 96.173 & 101.44 & 112.67 & 121.86 & 134.58 & 152.38 & 175.18 & 210.12 & 248.6 \\
\hline & Unidirectional & 95.059 & 101.29 & 109.73 & 119.54 & 138.58 & 164.28 & 187.28 & 221.15 & 266.51 & 321.41 \\
\hline & KB_Selective & 98.561 & 104.13 & 110.54 & 121.71 & 136.15 & 155.07 & 178.41 & 206.04 & 244.64 & 304.18 \\
\hline & LT_Selective & 96.43 & 100.04 & 107.08 & 116.84 & 133.09 & 150.69 & 170.3 & 195.46 & 229.83 & 278.42 \\
\hline
\end{tabular}

Due to increases in transmission and retransmission of packets with the simulation time collision increases. As the nodes in network is almost constant therefore collision ratio is directly depends only on number of collided packets in defined duration. Fig. 11 shows the collision ratio for selective time duration in 10 simulations and it is less for our proposed work as compared to others.

Average wait time in milliseconds has been recorded for various simulations. This delay is increases in each simulation for every method because the network congestion increases. Packet retransmission is one of the major reasons for same. Also increase in nodes increases the delay. Deviation in delay or wait time with the increase in simulation time is shown in Fig. 12.

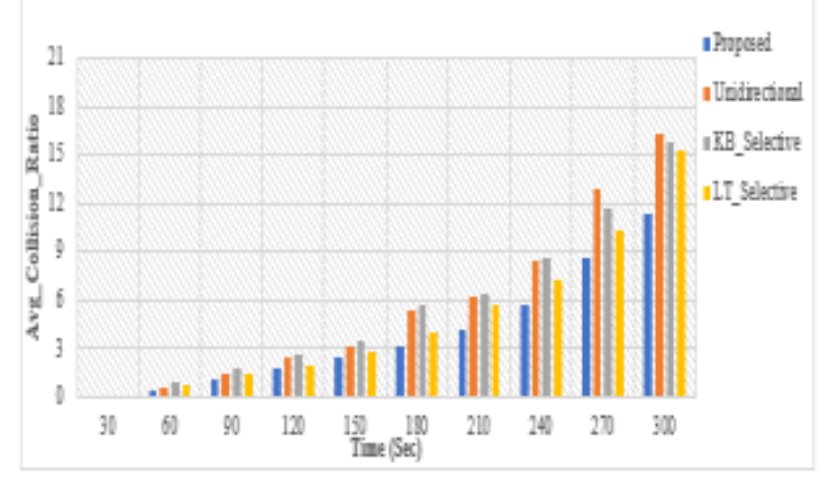

Fig. 11. Simulation Result for Collision Ratio Versus Time.

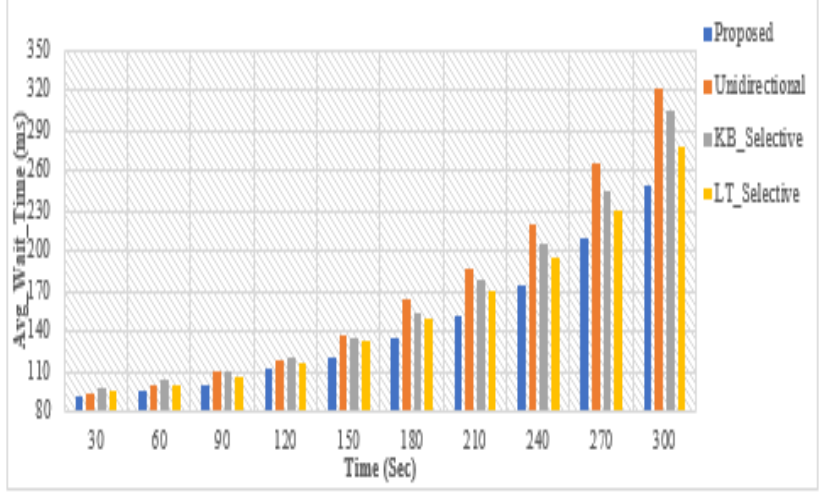

Fig. 12. Simulation Result for Wait Time Versus Time.

Further the analysis was done on the basis of node density in ad hoc network. Now we are varying the number of vehicles in the network from 40 to 400 vehicles per hour for simulation time. Recorded metrics are given in Table IV and performance comparisons for considered schemes are shown from Fig. 13 to 17.

With the increase in the node density in the network the average packet delivery ratio (PDR) is decreased. Reason for such type of changes in PDR is, data packets to be transmitted are increased with increase in number of vehicles. Tendency towards collision or loss of packets will increase which led to decrease in overall effective packet delivery ratio for simulation duration. Also in the proposed work PDR decreases but still it is performing much better as compared to others as shown in Fig. 13. 
TABLE IV. RESUlt TABLE WITH Node Density

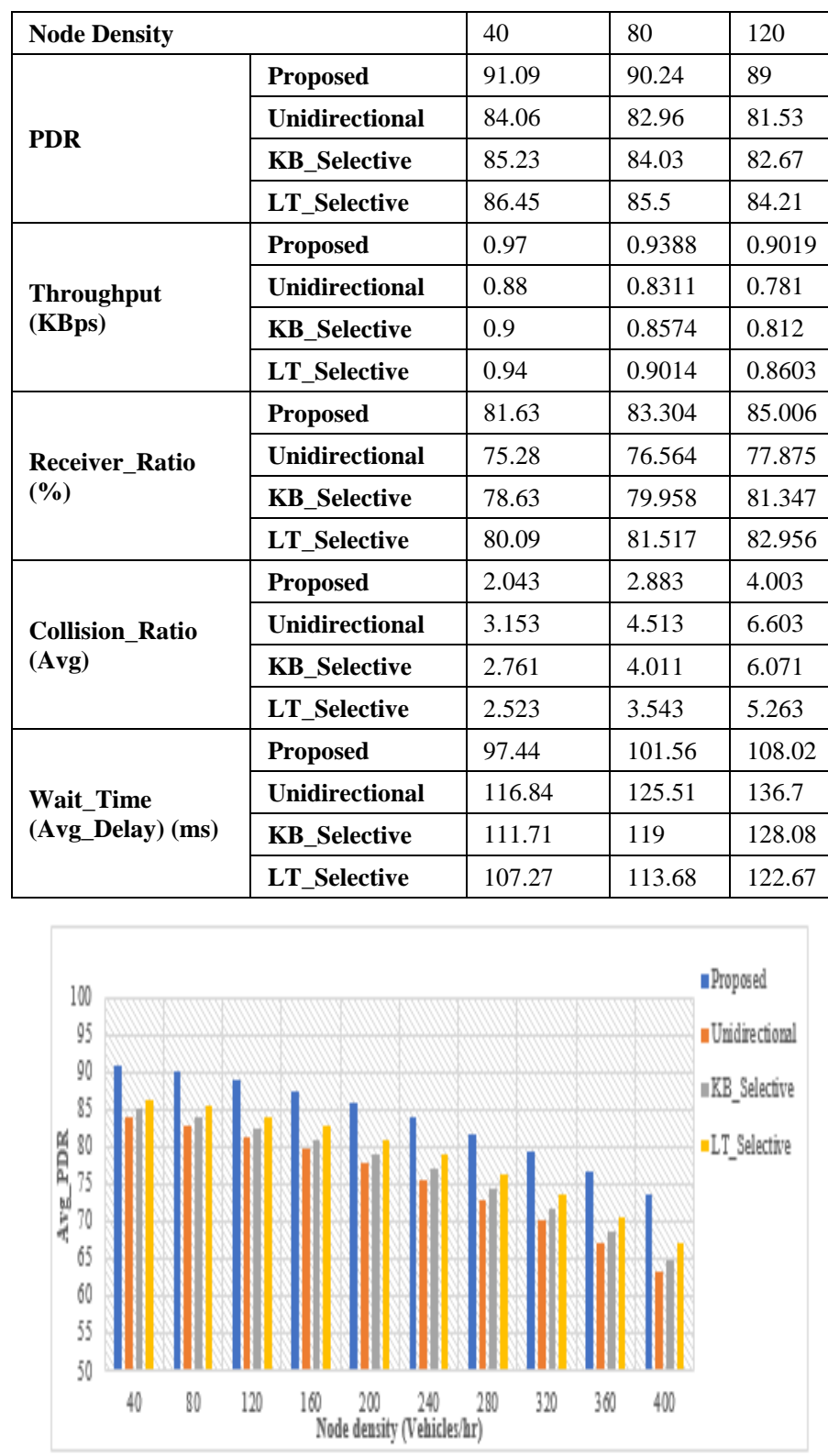

Fig. 13. Simulation Result for Packet Delivery Ratio versus Node Density.

With the increase in vehicle density, throughput of network is decreases because every node is not receiving the packets and also new nodes are continuously entering in network will cause the increase in network traffic for data packets. Therefore with the highest node density there will be least throughput. Our work is giving better result compared to other in simulation duration for network throughput with the variation in number of vehicles per hour as shown in Fig. 14.

Fig. 15 shows the fast coverage of information by proposed approach with the increase in node density compared to other approaches. Our methodology is delivering data packets by covering all the nodes in approximate $240 \mathrm{sec}$ while others not.

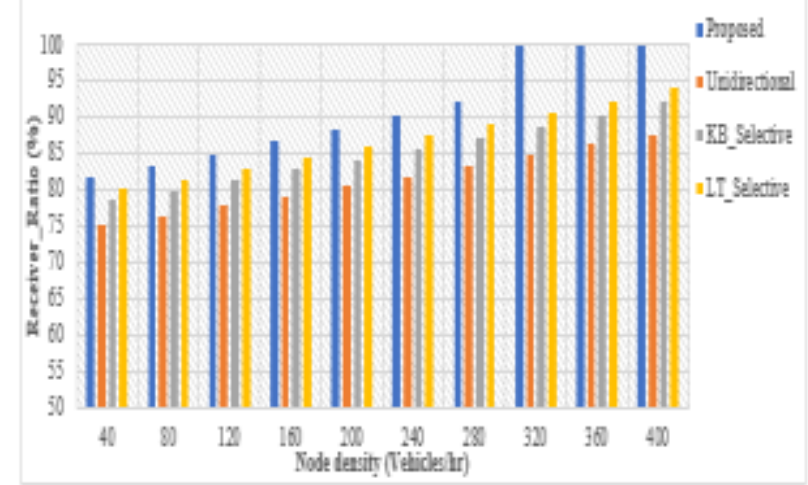

Fig. 15. Simulation Result for Receiver Ratio versus Node Density. 
It is obvious that number of collisions will increase with the increase in vehicle density but how much efforts can be applied to reduce the collision ratio is more important. A better performance of our algorithms in comparison to others on collision ratio with the increase in node density has been shown in Fig. 16. Initially the collision ratio is not increasing rapidly. When node density increases to around 300 vehicles per hour there is growth in collision ratio for all approaches but minimum for our proposed approach.

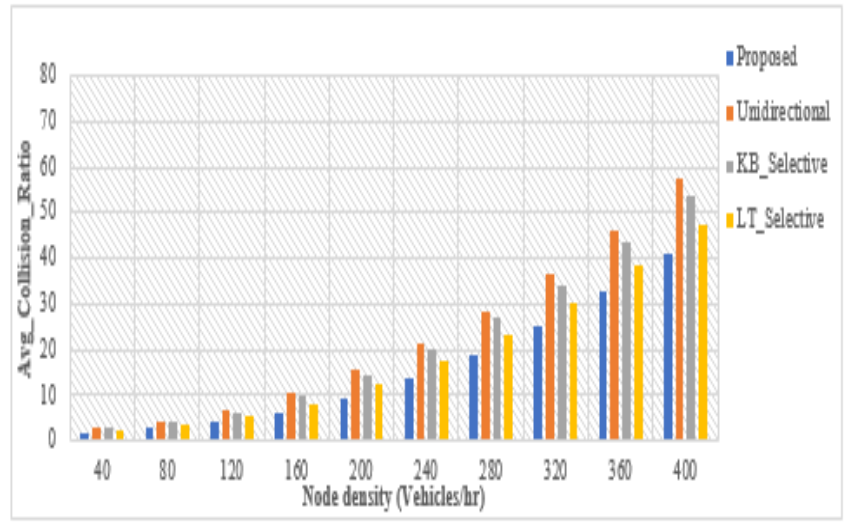

Fig. 16. Simulation Result for Collision Ratio versus Node Density.

Average delay for all simulations durations is increasing with the increase in node density of network. Average delay is approx. 90 milliseconds when we have taken minimum node density of 40 vehicles per hour. Up to density of 200 vehicles per hour, average delay is not increasing rapidly but after that it increases exponentially as shown in Fig. 17. Recorded values of simulations show the better performance for delay metrics in our proposed approach.

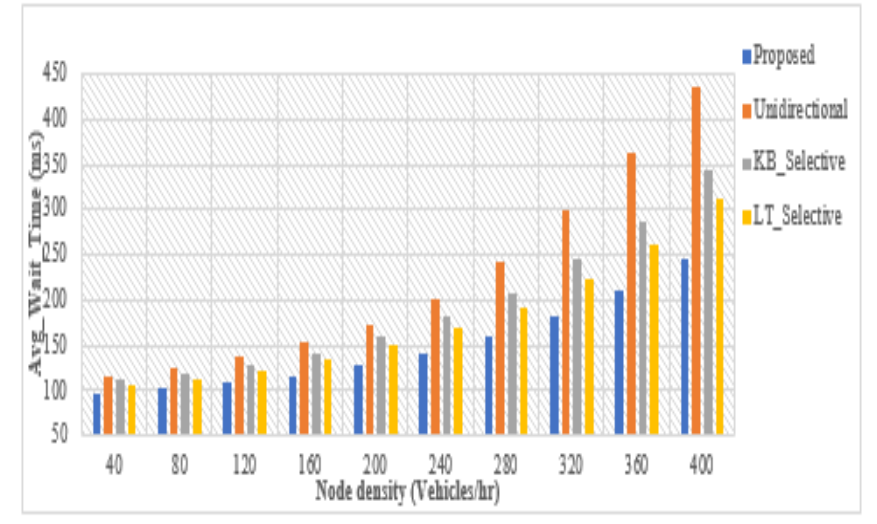

Fig. 17. Simulation Result for Wait Time versus Node Density.

\section{CONCLUSION AND FUTURE WORK}

In this article of proposed work for selective forwarders from 1-hop we can conclude that the performance for all metrics is better from other three approaches. Here the approach of choosing either farthest or behind the farthest node moving in similar direction based on their exit time calculation from source transmission range is providing effective reduction in overall delay and increase in throughput of network. Filtering of vehicles using region assignment also reducing the overall time taken by approach to select relay nodes. Overall transmission range of source is divided into regions and when vehicle entered in this range, it has been allotted to a particular region. During relay node selection if the vehicle belongs to rejection zones then it will not be the part of relay selection process. Therefore overhead for selection has been reduced effectively and this led to increase in system performance and approach is quite faster in relay node selection. Selective schemes for data dissemination give better results compared to unidirectional in the dense traffic. Various features of designed approach are, it covers the network rapidly, reducing the number of retransmissions and effectively reducing the average delay. Finally the proposed novel approach providing efficient and effective data dissemination in infrastructure less vehicle to vehicle communication.

Proposed technique of data dissemination can be further extended for comparison with some more type of similar approaches with different parameter values. Also the work can be extended to include the technique of cluster with head and members instead of regions creation and assignment. Proposed work may be examined for urban and infrastructure based scenarios with other parameters. Further scope in proposed approach to work on rank based message transmission where the priority can be assigned as per emergency, critical or important information.

\section{REFERENCES}

[1] R. Kumar and M. Dave, "A review of various VANET data dissemination protocols," International Journal of $u$ - and e-Service, Science and Technology, vol. 5, No. 3, , pp. 27-44, September, 2012.

[2] R. Meneguette, G. Maia, E. R. M. Madeira, A. R. Pinto, A. A. F Loureiro and L. A. Villas, "A new Solution to perform Data Dissemination in Vehicular Networks," Brazilian Symposium on Computer Networks and Distributed Systems, 2014, pp. 443-450. 10.1109/SBRC.2014.53.

[3] J.A Sanguesa, M. Fogue, P. Garrido, F. J. Martinez, J. C. Cano and C. T. Calafate, "A Survey and Comparative Study of Broadcast Warning Message Dissemination Schemes for VANETs", Mobile Information Systems, vol. 2016 , pp. $1-18,2016$. https://doi.org/10.1155/2016/8714142.

[4] M. Sichitiu and M. Kihl, "Inter-vehicle communication systems: a survey,".IEEE Communications Surveys \& Tutorials, vol.10, No. 2, , pp. 88-105, $2^{\text {nd }}$ Quarter, 2008. 10.1109/COMST.2008.4564481.

[5] Co-operative Networks for Intelligent Road Safety, [Online]. Available: https://trimis.ec.europa.eu/project/co-operative-networks-intelligentroad-safety.

[6] S. Allani, T. Yeferny, R. Chbeir, and S.B. Yahia, "A Novel VANET Data Dissemination Approach Based on Geospatial Data," Procedia Computer Science, vol. 98, pp. 572-577, 2016. https://doi.org/10.1016/j.procs.2016.09.089.

[7] W. Viriyasitavat, O. Tonguz and F. Bai, "UV-CAST: an urban vehicular broadcast protocol," IEEE Communications Magazine, vol. 49(11), pp. 116-124, Nov. 2011. 10.1109/VNC.2010.5698266.

[8] S. Allal and S. Boudjit, "Geocast Routing Protocols for VANETs: Survey and Guidelines," Sixth International Conference on Innovative Mobile and Internet Services in Ubiquitous Computing (IMIS), Palermo, Italy 2012, pp. 323-328. 10.1109/IMIS.2012.133.

[9] B. Alsubaihi and A. Boukerche, "Semantic and Self-Decision Geocast Protocol for Data Dissemination over VANET (SAS-GP)," IEEE Wireless Communications and Networking Conference (WCNC), New Orleans, LA, USA, 2015, pp. 1948-1953. 10.1109/WCNC.2015.7127766.

[10] F. Cunha, G. Maia, A. Viana, R.A. Mini, L. Villas and A.A. Loureiro, "Socially Inspired Data Dissemination for Vehicular Ad Hoc Networks," Proceedings of the 17th ACM International Conference on 
Modeling, Analysis and Simulation of Wireless and Mobile Systems, MSWiM '14, ACM, New York, 2014, pp. 81-85. https://doi.org/10.1145/2641798.2641834.

[11] M. Bilal, P. M. L. Chan and P. Pillai, "Fastest-Vehicle Multi-hop Routing in Vehicular Ad hoc Networks," Proc. of 10th IEEE Int'l Conf. on Computer and Inf. Tech. (CIT), Bradford, UK , 2010, pp. 773-778. 10.1109/CIT.2010.148.

[12] I. Achour, T. Bejaoui, A. Busson and S. Tabbane, "SEAD: A Simple and Efficient Adaptive Data Dissemination Protocol in Vehicular Adhoc Networks," Wireless Network 22, pp. 1673-1683, 2016. https://doi.org/10.1007/s11276-015-1050-9.

[13] R. Kumar and M. Dave, "A comparative study of Various Routing Protocols in VANET, " International Journal of Computer Science Issues (IJCSI), vol. 8, Issue 4, No.-4, pp. 643-648, July 2011.

[14] B. Bako, and M. Weber, "Efficient Information Dissemination in VANETs," Advances in Vehicular Networking Technologies, Dr Miguel Almeida (Ed.), ISBN: 978-953-307-241-8, April 2011, pp. 4564. 10.5772/15533.

[15] F. J. Ros, P. M. Ruiz PM and I. Stojmenovic, "Acknowledgment-Based Broadcast Protocol for Reliable and Efficient Data Dissemination in Vehicular Ad Hoc Networks". IEEE Transactions on Mobile Computing, vol. 11(1).1, pp. 33-46, Jan. 2012. 10.1109/TMC.2010.253.

[16] B. Tian, K.M. Hou, and J. Li, "TrAD: Traffic Adaptive Data Dissemination Protocol for Both Urban and Highway VANETs," in IEEE, 30th International Conference on Advanced Information Networking and Applications (AINA), Crans-Montana, Switzerland, 2016, pp. 724-731. 10.1109/AINA.2016.20.

[17] M. A. Leal, M. Rockl, B. Kloiber, F. de. P. Muller and T. Strang, "Information-centric opportunistic data dissemination in Vehicular Ad Hoc Networks".13th International IEEE Annual Conference on Intelligent Transportation Systems, Funchal, Portugal, September 19-22, 2010, pp. 1072-1078. 10.1109/ITSC.2010.5625053.

[18] T. Nikolovski, and W.R. Pazzi, "Delay Tolerant and Predictive Data Dissemination Protocol (DTP-DDP) for urban and highway vehicular ad hoc networks (VANETs),". Proceedings of the 6th ACM Symposium on Development and Analysis of Intelligent Vehicular Networks and Applications - DIVANet '16, Malta, Malta, November 13-17, 2016, pp. 67-74. https://doi.org/10.1145/2989275.2989280.

[19] M. Bakhouya, J. Gaber, J., and P. Lorenz, "An adaptive approach for information dissemination in Vehicular Ad hoc Networks," Journal of Network and Computer Applications, vol. 34(6), pp. 1971-1978, Nov 2011. https://doi.org/10.1016/j.jnca.2011.06.010

[20] J. B. D. da Costa, A. M. de Souza, D. Rosário, E. Cerqueira and A. V. Leandro, "Efficient data dissemination protocol based on complex networks' metrics for urban vehicular networks," Journal of Internet Services and Applications, pp. 10-15, 2019. https://doi.org/10.1186/s13174-019-0114-y.
[21] M. Ali, A. W. Malik, A. U. Rahman, S. Iqbal, and M.M. Hamayun, "Position-based emergency message dissemination for Internet of vehicles," International Journal of Distributed Sensor Networks, vol. 15(7), pp. 1-12, June 2019. 10.1177/1550147719861585.

[22] J. Costa, D. Rosario, A. M. de Souza, L. A Villas and E. Cerqueira, "Data Dissemination Based on Complex Networks' Metrics for Distributed Traffic Management Systems," IEEE Symposium on Computers and Communications (ISCC), Natal, Brazil, 2018, pp. 01062-01067. 10.1109/ISCC.2018.8538601.

[23] J. Sospeter, D. Wu, S. Hussain and T. Tesfa, "An Effective and Efficient Adaptive Probability Data Dissemination Protocol in VANET," Data, MPDI, Vol. 4(1), 1, 2019. 10.3390/data4010001.

[24] R. Oliveira, C. Montez, A. Boukerche and M. S. Wangham, "Reliable Data Dissemination Protocol for VANET Traffic Safety Applications", Ad Hoc Networks, vol. 63, pp. 30-44, Aug 2017. 10.1016/j.adhoc.2017.05.002.

[25] A. Baiocchi, P. Salvo, F. Cuomo, I. Rubin, "Understanding Spurious Message Forwarding in VANET Beaconless Dissemination Protocols: An Analytical Approach," IEEE Transactions on Vehicular Technology, Vol. 65 (4), pp. 2243-2258, 2016. 10.1109/TVT.2015.2422753.

[26] L. Liu, C. Chen, T. Qiu, M. Zhang, S. Li and B. Zhou, "A data dissemination scheme based on clustering and probabilistic broadcasting in VANETs," Vehicular Communications, vol. 13, pp.78-88, July 2018. 10.1016/j.vehcom.2018.05.002.

[27] M. Chaqfeh, H. El-Sayed and A. Lakas, "Efficient data dissemination for urban vehicular environments," IEEE Transactions on Intelligent Transportation Systems, vol. 20, no. 4, pp.1226-1236, Aug 2018. 10.1109/TITS.2018.2850068.

[28] D. G. Reina, V. Sharma, I. You and S. Toral, "Dissimilarity metric based on local neighboring information and genetic programming for data dissemination in vehicular ad hoc networks (VANETs)," Sensors, Vol. 18, No. 7, pp.1-18, 2018. 10.3390/s18072320.

[29] K. N. Qureshi, H. Abdullah, F. B. Hussain, S. and K. Awan, "Clusterbased data dissemination, cluster head formation under sparse, and dense traffic conditions for vehicular ad hoc networks," International Journal of Communication Systems, vol. 31, no. 8, pp. 1-16, 2018. 10.1002/dac.3533.

[30] M. U. Farooq, K. U. R Khan and S. Mohammed, "Selective flooding techniques for dissemination in VANETs," IEEE International Conference on Contemporary Computing and Informatics (IC3I), Noida, India, 2016, pp.369-374. 10.1109/IC3I.2016.7917991

[31] R. Pradhan and T. De, "A selective forwarding technique for data dissemination in vehicular ad hoc networks based on traffic parameters," International Journal of Ad Hoc and Ubiquitous Computing, vol. 35, no. 4, pp.179-190 2020. 10.1504/IJAHUC.2020.111460. 